\title{
MULTIPLE-APERTURE OPTICAL TELESCOPES: COPHASING SENSOR TESTBED
}

\author{
B. Sorrente, F. Cassaing, F. Baron, C. Coudrain, B. Fleury, F. Mendez, L. Mugnier, V. Bentadj-Paris, V. Michau, \\ J. Montri, G. Rousset, L. Rousset-Rouvière, and M.-T. Velluet
}

ONERA/DOTA/CC, BP 72, 92322 Châtillon CEDEX, France. E-mail: Beatrice.Sorrente@onera.fr

\begin{abstract}
The goal of a cophasing sensor (CS) is to measure the phase disturbances between the sub-apertures or inside each aperture of a telescope. Three CSs are currently studied at ONERA. A first CS for Earth imaging is based on phase diversity on extended sources (cf companion paper by L. Mugnier). A second CS for intercalibration uses phase retrieval on a point source. The third CS for nulling interferometry ("DWARF", for the ESA/DARWIN mission) is based on similar algorithms.

To test performance of these CSs, ONERA has defined and integrated a multipurpose bench, "BRISE". Its main features are the simultaneous imaging of a point source and of an extended source, the minimisation of absolute and differential disturbances, the use of any aperture configuration and the generation of pure calibrated piston/tip/tilt aberrations on three sub-apertures by a dedicated PZT-based device. Preliminary experimental results are consistent with numerical simulations and confirm nanometric performance.
\end{abstract}

Key words: Earth observation; Interferometry; Synthetic aperture optics; Phased array imaging systems; Cophasing sensor; Phase measurement; Phase diversity.

\section{CONTEXT}

Multiple Aperture Optical Telescopes (MAOT) are considered for high-resolution spaceborne missions such as Earth imaging (cf companion paper [1]) or astronomy (NGST or ESA/DARWIN). To perform real-time correction of mechanical disturbance in these MAOTs deployed after launch, a critical component is the Cophasing Sensor (CS), whose goal is to measure the relative positioning (piston/tip/tilt error) of the MAOT sub-apertures, or even the higher-order aberrations on each sub-aperture.

Three CSs are currently investigated at ONERA. The first one is a Phase Diversity Sensor (PDS) for Earth imaging [1]. To validate piston estimation from extended scenes, a Phase Retrieval Sensor (PRS) is operated in parallel on a reference unresolved source. The third CS, DWARF (DarWin AstRonomical Fringe sensor), is a PDS/PRS developed for ESA by KayserThrede/ONERA/Alcatel Space and defined by ONERA $[2,3]$.

To test performance of these CSs, ONERA has designed in 2002 and built in 2003 a testbed for the accurate generation of multiple-aperture phase perturbation, using experience from a previous bench [4] and stellar interferometry from the ground [5]. This bench, BRISE (Banc Reconfigurable d'Imagerie sur Scènes Etendues), was designed in the framework of the Earth imaging study and includes a PDS and a PRS [1]. While latest adjustments are performed, BRISE is operated since January 2004 and first results are just available. BRISE was designed as open as possible, allowing DWARF to be tested on BRISE by ONERA in March.

BRISE is described in section 2. Section 3 deals with the PRS, which can be only operated in restricted cases. The more complex PDS, for full phase estimation on any scene, is addressed in section 4. In each case, the first subsection describes the principle and the second subsection gives results from simulations or first experiments.

\section{THE BRISE BENCH}

\subsection{Description}

A schematic view of BRISE is shown in Fig. 1. BRISE is mainly composed of four sub-systems, detailed herebelow: the source module delivers two objects, an extended scene EXT and a reference point source REF; the detection module records focal-plane images of the two objects and implements a PDS; the perturbation module images the source on the detector, defines the aperture configuration and introduces calibrated aberrations; the control module drives the experiment. Afocal input/output ports are included, to host "visitor instruments" such as DWARF or our Zygo interferometer.

Special care has been given to the control of errors that could limit CS performance or their estimation. The two objects EXT and REF are observed simultaneously through very close paths, to minimise the effect of field 


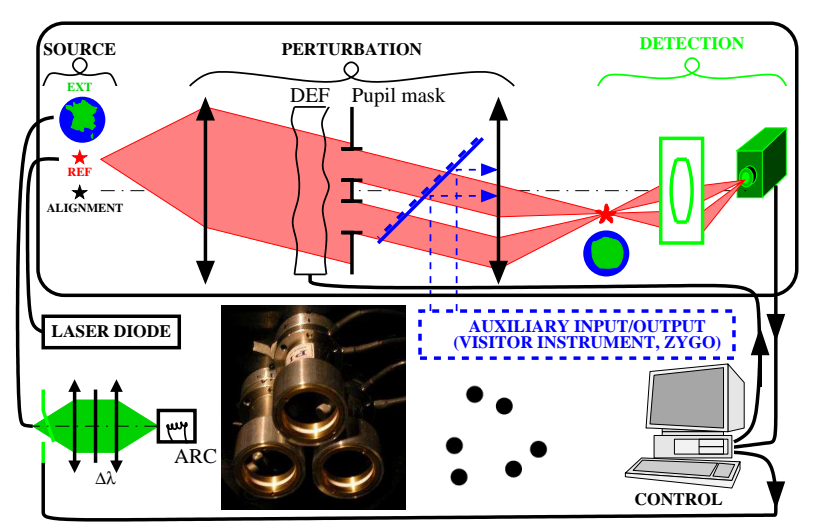

Figure 1. Schematic view of BRISE and photograph of the deformable mirror (DEF).

aberrations, vibrations or air turbulence. A very accurate OPD calibration can thus be achieved thanks to the high SNR of the REF measurement. To reduce air turbulence or temperature-induced drifts, BRISE is enclosed inside a wooden hut located in a temperature-controlled room. An additional metallic baffling on the bench minimises volume and temperature gradients around the beams. Active pneumatic supports isolate BRISE from vibrations.

The main characteristics of BRISE are summarised in Table 1 . The spectral band is currently set by the refractive components in the detection module optimised in the $[0.55 ; 0.83] \mu \mathrm{m}$ range, but the all-reflective bench can be operated in a much larger domain.

Table 1. Main current characteristics of BRISE.

\begin{tabular}{|l|r|l|}
\hline Parameter & Value & Unit \\
\hline Full spectral band & {$[0.4 ; 1]$} & $\mu \mathrm{m}$ \\
\hline Reference source wavelength & 0.635 & $\mu \mathrm{m}$ \\
\hline Field of the extended object & $\simeq 250$ & pixels \\
\hline Maximal aperture diameter & $B \simeq 60$ & $\mathrm{~mm}$ \\
\hline Aperture configuration & $3 \times(D=20)$ & $\mathrm{mm}$ \\
\hline Perturbation amplitude & $\simeq \pm 4$ & $\mu \mathrm{m}$ \\
\hline Total camera field & $1317 \times 1035$ & pixels \\
\hline Beam height & 160 & $\mathrm{~mm}$ \\
\hline
\end{tabular}

\subsubsection{The source module}

An extended object and a reference point are put side by side in the focal plane of the perturbation module by a roof-top mirror. The two light sources are located outside the hut to avoid environmental disturbances. Small optical benches are used to couple the sources in optical fibres delivering light in the baffling.

The extended object (EXT) is an Earth scene (Fig. 2) or an astronomical object on a photographic plate. The output of the multimode fibre fed by an arc lamp is re-imaged on the object. The spectral band and the intensity can be simply selected by filters inserted by the operator on the external benches.
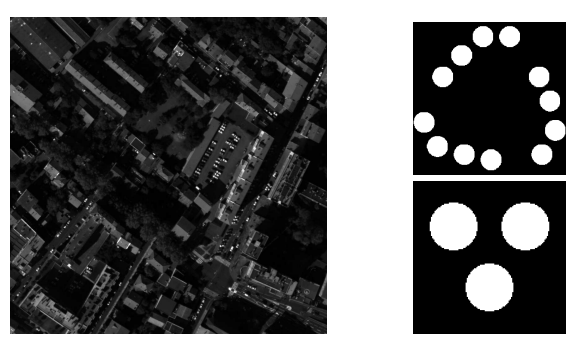

Figure 2. Left: extended scene used for simulations and experiments. Right: masks with 3 and 12 sub-apertures.

The reference object (REF) is the direct output of the monomode fibre, fed by a fibred laser diode operating at $635 \mathrm{~nm}$ or the arc lamp.

\subsubsection{The perturbation module}

The design of this module is driven by the deformable mirror (DM) used to introduce phase perturbations. For multiple-aperture CS considered here, the DM must include a calibrated piston/tip/tilt generator with a stroke of several wavelengths. Usual DMs with a continuous surface deformed by PZT actuators can not introduce a pure piston on a sub-aperture without changing the high order modes and are affected by PZT drift. We have thus manufactured a specific segmented DM (DEF hereafter) with three planar mirrors of diameter $21 \mathrm{~mm}$ (Figs. 3 and 1). Two mirrors are mounted on Physik Instrument piston/tip/tilt S-316.10 platforms, the third on a dummy support in the same material. Each platform has three $120^{\circ}$ PZT motors with nanometric closed-loop control on an internal strain gauge. The PZT stroke, about $12 \mu \mathrm{m}$, being much smaller than the mechanical dispersion on the total height of the platform, intermediate manuallypolished wedges and tinsels have been inserted between the platforms and the supporting plate to pre-align the three sub-apertures within the PZT stroke (Fig. 3).

The DM is in autocollimation to ensure circular apertures. A planar mirror is also available for full-aperture measurements without perturbation. A pupil mask is used to define uniquely the aperture configuration (position and diameter $D$ of the sub-apertures). Fig. 2 shows the most used mask, with three $D=20 \mathrm{~mm}$ sub-apertures, and the 12 sub-aperture mask from the Earth imaging study [1]. The collimated beam can be sent to (or input from) an external device, such as another CS or a reference interferometer.

The diameter $(B=60 \mathrm{~mm}$ ) of DEF sets the focal length of the collimating and focusing optics of the perturbation module. The autocollimation creates an image focalplane near the source with magnification -1 , which is routed to the detection module by a folding mirror. 


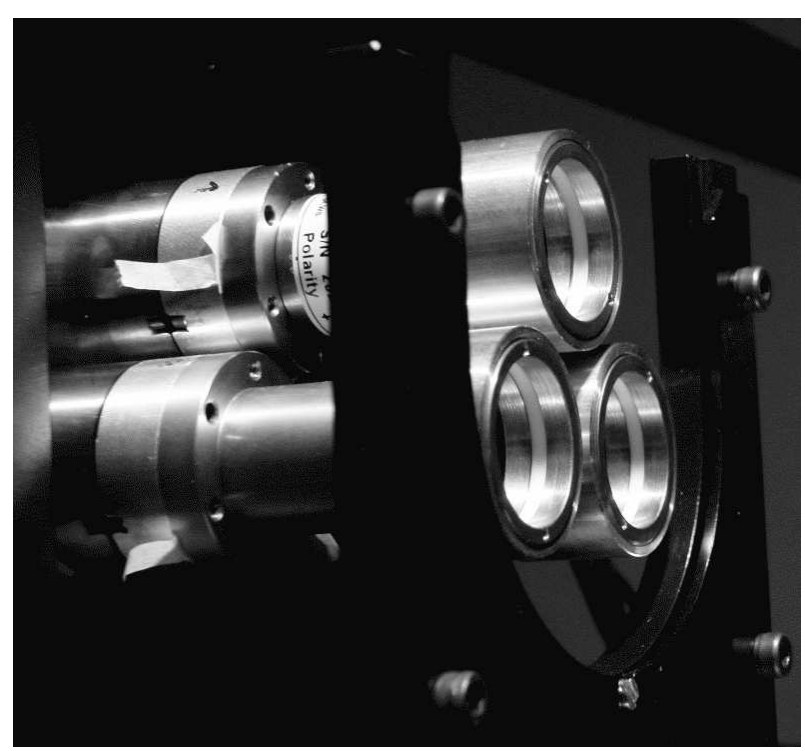

Figure 3. The DM mirror currently mounted on BRISE and the aperture mask support.

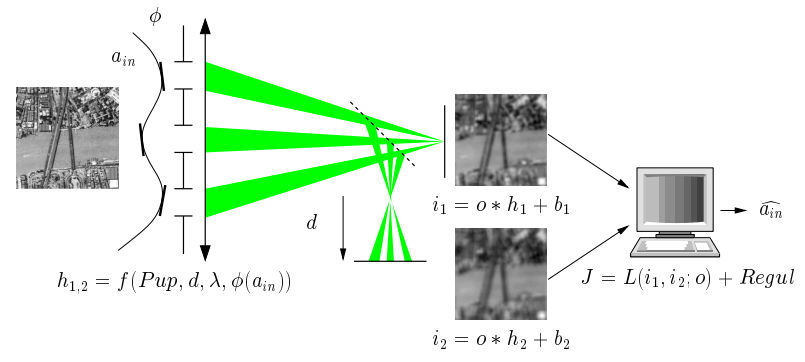

Figure 4. Principle of a PDS implemented near a MAOT focal-plane.

\subsubsection{The detection module}

This module is based on a Roper camera with a $6.8 \mu \mathrm{m}$ pitch. To correctly sample the image, an optical relay re-images the output of the perturbation module on the camera with a small magnification. An additional lens can be inserted to re-image the pupil on the camera for alignment. The camera shutter is used during integration, but it is foreseen to replace it by similar shutters mounted on the external source benches to avoid vibrations on BRISE.

The sensor selected for Earth observation is a PDS [1]. This technique detailed in section 4 is based on the joint analysis of a focal-plane focused image and a focal-plane slightly-defocused image (Fig. 4). Therefore, a PDS has been included in the BRISE detection module. An internal CS is therefore always available on BRISE, to control the DM shape when feeding another CS for instance.

The focused and defocused images are simultaneously recorded on the same CCD thanks to a beam-splitter and folding mirrors in the relay optics. The defocus is manually tuned by a micrometric translation stage. The $1317 \times 1035$ pixel camera field is large enough to simultaneously collect the focused and defocused images of the
EXT and REF objects, as illustrated on Fig. 5.

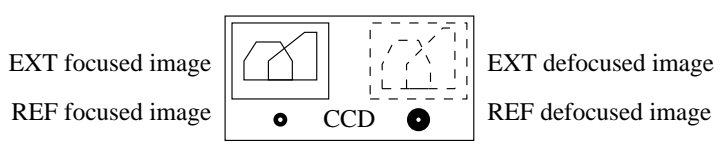

Figure 5. Schematic view of BRISE image focal plane.

\subsubsection{The control module}

The main goal of the control module is to synchronously drive the DM and the camera. Three computers on an Ethernet network are used (Fig. 6), with an hardware trigger. One PC controls the DM motion via six voltages. The second $\mathrm{PC}$ is dedicated to the image acquisition of the detection module. Both PCs implement low-level drivers under $\mathrm{C} /$ Windows and a client/server architecture. Data are transfered to the Unix work station and stored on a local disk with external backup through the LAN.

A Tcl/Tk Graphical User Interface is used to set the main experiment parameters (exposure time, windowing, number of images,...) and to prompt the user for additional inputs (object, mask, filter, etc). All the configuration is stored in a log file with each data file for later reference.

Two operating modes are supported. In the measurement mode, data are written on disk and processed offline. An IDL tool has been written to automatically process images from their log file. In the command mode, a few pre-defined DM modulation patterns are applied and IDL is automatically run after image acquisition to derive, from the images, the incremental voltages to apply on the DM. The few algorithms and modulation patterns available make this one-step closed-loop mode very useful.

\subsection{First results obtained with BRISE}

The most critical issue during BRISE manufacturing was the cophasing of DEF. The differential height of the three wedges had to be manually adjusted to better than $\pm 1,5 \mu \mathrm{m}$ to ensure a sufficient PZT stroke on both sides of the aligned position. A procedure based on measurements in the Michelson interferometer has been defined

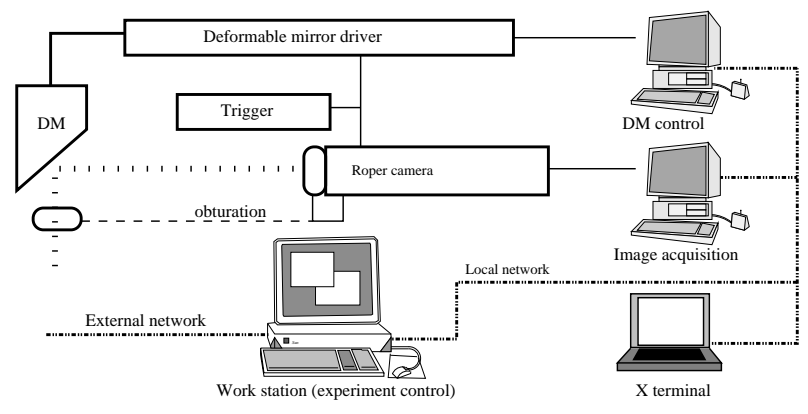

Figure 6. Synoptic of the hardware architecture. 
and successfully performed. Fig. 3 shows the micrometric tinsels inserted following the $120^{\circ}$ geometry.

Fig. 7 shows an experimental focused image of REF with DEF roughly aligned. Although the REF fibre is slightly resolved by the perturbation module, we will assume for simplicity that this image is the bench PSF. The Fourier Transform of this image, the OTF, shows the expected central peak (sum of the autocorrelation of each aperture) and the six satellite fringe peaks produced by the three baselines of the aperture. The amplitude of the fringe peaks is smaller than in the theoretical MTF (1/3 of the central peak) since REF is resolved.

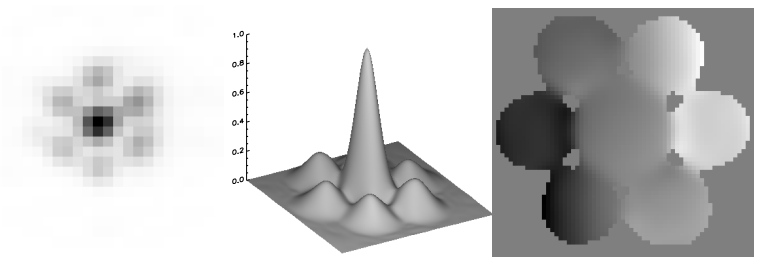

Figure 7. Experimental PSF acquired on BRISE (left) and its Fourier transform (center: modulus, right: phase computed where the modulus is significant).

The first quantitative measurement made is to estimate BRISE's stability. A set of 128 images of REF has been acquired using the laser diode and the reference mirror, every 3 seconds with a 20 ms exposure time. Fig. 8 shows the temporal evolution of the differential piston estimated with the algorithm described in section 3.1. The differential pistons show a slow drift of amplitude $7.5 \mathrm{~nm} / \mathrm{min}$, while the closure phase computed from the three subapertures is perfectly stable, with a $0.6 \mathrm{~nm}$ repeatability $(\lambda / 1000)$. This first measurement confirms that nanometric measurements are possible on BRISE, at least on short time-scales. The slow drift shows a priori that thermal equilibrium is not reached. Assuming a coefficient of thermal expansion equal to $210^{-5}$, this corresponds to a $0.4 \mathrm{mK} / \mathrm{min}$ drift which is very likely since although the camera water cooling has been recently installed, the fan is not yet unplugged and may have increased temperature in the baffling when the camera was turned on. Future BRISE measurements will teach us more about its environment and we expect that the last foreseen upgrades will allow to lower these drifts.

\section{PHASE-RETRIEVAL COPHASING SENSOR}

Phase estimation from focal-plane data (or phase retrieval) suffers from phase ambiguity. However, with a non-redundant MAOT and for a point source, the main modes of interest (the sub-aperture piston/tip/tilt) can be uniquely estimated. Fig. 7 shows that, the phase perturbation being small, the PSF is nearly centro-symmetric. However, the OTF phase is clearly affected by piston/tip/tilt. Since the OTF phase derives simply from the sub-aperture phases, an estimator of piston/tip/tilt on each aperture can be derived from the analysis of the sole focal-plane data $[6,7]$.

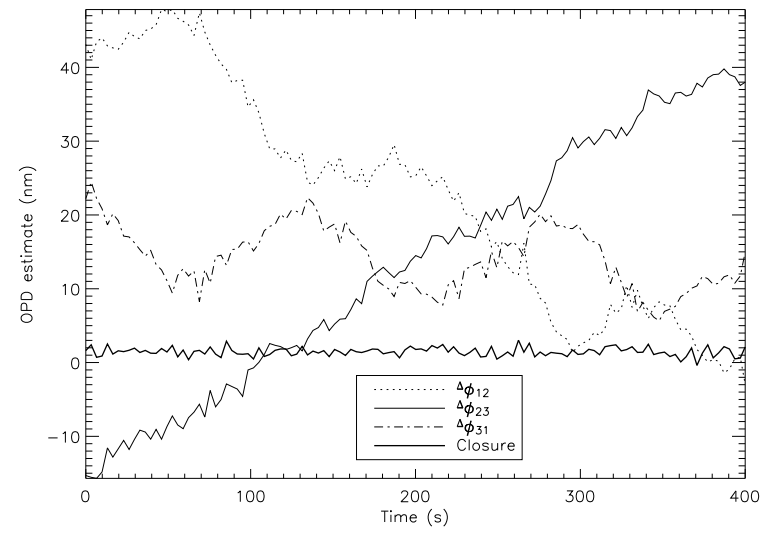

Figure 8. Temporal evolution of the differential pistons (arbitrary origin) and of the phase closure.

\subsection{Principle of phase-retrieval}

A piston on one sub-aperture has a simple effect on the OTF, merely a phase offset on each inter-correlation peak involved. Deriving the piston from the focal-plane image is an inverse problem, that can be solved by minimising a criterion $J$ equal to the distance between the recorded image $i_{1}$ and the image expected from the known object $o$ and the PSF $h_{1}$ derived from the unknown phases $a_{i, n}$ where $i, n$ are the Zernike and aperture indexes (Fig. 4). Statistical properties of the noise $b_{1}$ can also be included in $J$. This problem has an analytical solution and a linear piston estimator can be derived for a stationary white Gaussian noise [8]. For $N_{T}$ sub-apertures, the piston estimate for aperture $m$ is given by:

$$
a_{1 m}=\frac{1}{N_{T}} \sum_{n=1, n \neq m}^{N_{T}} \operatorname{Arg}\left\{\int_{D_{n m}} \operatorname{FT}(i) \operatorname{FT}(o)^{*} \Lambda\right\}
$$

where $\Lambda$ is the modulus of an unaberrated OTF peak and $D_{n m}$ is the support of the inter-correlation peak of apertures $n$ and $m$. Eq. (1) can be read as follows: for an unresolved source $(\mathrm{FT}(o)=1)$, the piston for any sub-aperture $m$ is the average of the differential pistons measured on all baselines involving that sub-aperture, those pistons being measured by averaging the OTF FT $(i)$ over each peak. The main advantage of the inverse problem approach is to give the weighting factor (the fringe peak modulus $\Lambda$ ) minimising noise propagation when averaging over $D_{n m}$ with a variable signal-to-noise ratio.

For tip/tilt estimation, a similar algorithm can be derived for small aberrations if $N_{T} \geq 3$. The tilt on each aperture is derived from differential tip/tilts estimated by a weighted averaging over the fringe peaks and the average tilt obtained on the central peak.

\subsection{Performance of the phase-retrieval CS}

Performance of the PRS has been computed by simulation. A set of 100 images has been generated with the 3 sub-aperture configuration (Fig. 2). Photon noise is 
added to the PSF as a Poissonian noise and the Read Out Noise $(\mathrm{RON}=20)$ is simulated by a Gaussian stationary noise. The number of photons is converted in DARWIN magnitudes ( $\mathrm{V}$ band).

Results are plotted in Fig. 9, which shows that RON is negligible before the limiting magnitude, which is 12.5 for piston and 10 for tip/tilt. The ESA target (mag. 12) can be reached by increasing the spectral band.

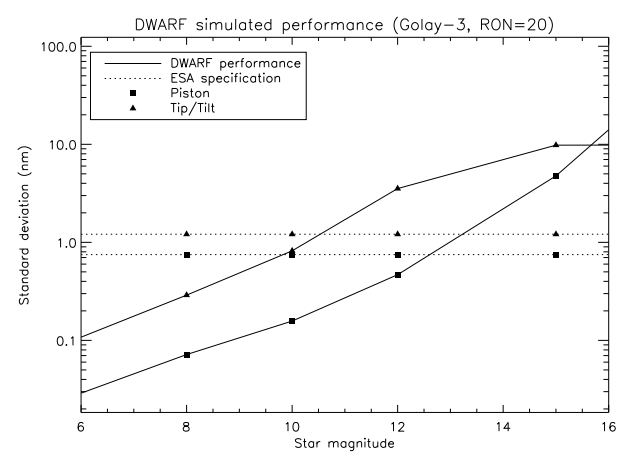

Figure 9. PRS performance for piston and tip/tilt.

A preliminary analysis of experimental results shown in Fig. 8 can be performed with Fig. 9. The average number of photons per frame, $N \simeq 1.110^{5}$, corresponds to a 11.7 magnitude. The $0.6 \mathrm{~nm}$ rms fluctuation of the experimental phase closure is thus in line with the simulated CS performance of $0.4 \mathrm{~nm}$. In addition, this performance is close to the quantum limit $\lambda / 2 \pi / \sqrt{N}=0.3 \mathrm{~nm}$ [9].

\section{PHASE-DIVERSITY COPHASING SENSOR}

In a more general case, when observing an unknown object $o$ or estimating Zernike modes of index $i \geq 4$, phaseretrieval is not possible from sole focal-plane data.

\subsection{Principle of phase diversity}

Unless one has strong structural information about the phase and/or about the object, the lack of information considerably limits the performance of phase-retrieval methods. Phase diversity is a technique that was proposed to add information about the unknown phase [10, 11]. The idea is to collect at least one additional image, which differs from the focused image by a known phase variation (cf Fig. 4). A simple way to introduce the known aberration is to use a beam splitter and two detectors, one of which being defocused.

This technique has been successfully used by many authors for wavefront sensing [12, 13, 14, 15, 16, 17] or for object restoration, as in solar imaging through turbulence $[18,19]$. It uses the optically simple wavefront sensor which consists in the imaging camera itself, but it requires a numerical and iterative processing to restore the unknowns from the images.
A recent PDS improvement is the marginal estimator developed at ONERA [15, 17]. The marginal approach, consisting in integrating the object out of the problem to perform only an aberration estimation, gives better results on monolithic telescopes at low SNR than the most widespread joint restoration of the object and the aberrations from focal-plane data. This result has also been verified for MAOTs [20].

\subsection{Performance of the phase diversity CS}

The internal PDS in the detection module has not been tested yet and the PDS implemented in DWARF to measure high-order aberrations (11 first Zernike modes on each telescope) will arrive in March. Therefore, only simulation results are available.

Fig. 10 shows the PDS performance for piston and tip/tilt with the two aperture configurations plotted in Fig. 2 and 100 noise outcomes. The source is the $256 \times 256$ pixels Earth scene plotted in Fig. 2. The phase diversity is a defocus, with an optimised amplitude of 1 (resp. 4) radian (rms, full aperture) for 3 (resp. 12) sub-apertures. For the same total flux $N=1.110^{5} \mathrm{ph}$, the extrapolated $5.5 \mathrm{~nm}$ piston error is much larger than on Fig. 9 since the visibility of the extended scene is much smaller. But cophasing requirements for imaging are less severe than for nulling, and the field can be increased for a better estimate.

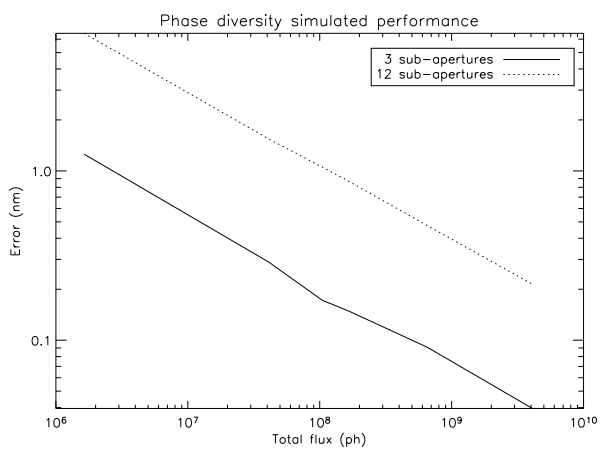

Figure 10. Total piston error for the two aperture configurations shown in Fig. 2.

\section{CONCLUSION}

The BRISE bench is almost complete. The long and difficult alignment of the segmented mirror, used to introduce calibrated pure piston/tip/tilt aberrations on two sub-apertures, has been successful. The bench is now producing interferometric measurements with three subapertures. First results show that the nanometric accuracy goal is reached.

Two kinds of focal-plane CSs will be tested on BRISE. Phase retrieval CSs use only focal-plane data and a linear algorithm, whereas phase diversity CSs use two different planes and iterative algorithms. Preliminary tests with the phase retrieval CS show that experimental and simulated results agree and are close to the quantum limit. 
Further investigation and additional results will be presented at the conference. If experimental results confirm the applicability of phase diversity for Earth observation, other data-processing algorithms we have in mind could be investigated for real-time operation. However, these promising results show that solutions with simple optical devices exist to cophase MAOTs with challenging requirements, for Earth imaging or nulling interferometry.

BRISE can be easily used for other purposes: the number of beams can be tuned from 1 to any value by changing the aperture mask, and most full-aperture deformable mirrors can be used. BRISE is open to any visitor CS fitting in $100 \times 70 \mathrm{~cm}^{2}$ (without the Zygo interferometer).

\section{ACKNOWLEDGEMENTS}

We thank DGA/SPOTI, CNES and ONERA for supporting the development of BRISE and the two sensors in the detection module. We thank ESA for supporting the development of DWARF and Kayser-Threde for the manufacturing of the DWARF breadboard.

\section{REFERENCES}

1. L. Mugnier, F. Cassaing, B. Sorrente, F. Baron, M.T. Velluet, V. Michau, and G. Rousset. Multipleaperture optical telescopes: some key issues for Earth observation from a GEO orbit. In 5th International Conference On Space Optics, volume SP-554, Toulouse, France, 2004. CNES/ESA, ESA.

2. F. Cassaing, F. Baron, E. Schmidt, S. Hofer, L. M. Mugnier, M. Barillot, G. Rousset, T. Stuffler, and Y. Salvadé. DARWIN Fringe Sensor (DWARF): Concept study. In Towards Other Earths, volume SP-539. ESA, 2003. Conference date: April 2003.

3. E. Schmidt, F. Cassaing, S. Hofer, M. Barillot, F. Baron, L. M. Mugnier, G. Rousset, and T. Stuffler. DARWIN Fringe Sensor (DWARF): Breadboard development. In Towards Other Earths, volume SP539. ESA, 2003. Conference date: April 2003.

4. M.-T. Velluet, V. Michau, and G. Rousset. Wavefront sensors for the active control of earth observation optical instruments. In International Conference on Space Optics, Toulouse (France), December 1997. CNES.

5. B. Sorrente, F. Cassaing, G. Rousset, S. RobbeDubois, and Y. Rabbia. Real-time optical path difference compensation at the Plateau de Calern I2T interferometer. Astron. Astrophys., 365:301-313, 2001.

6. F. Cassaing. Analyse d'un instrument à synthèse d'ouverture optique : méthodes de cophasage et imagerie à haute résolution angulaire. $\mathrm{PhD}$ thesis, Université Paris XI Orsay, December 1997.

7. F. Cassaing, L. Mugnier, G. Rousset, and B. Sorrente. Éléments-clés de la conception d'un instrument spatial à synthèse d'ouverture optique. In International
Conference on Space Optics, Toulouse (France), December 1997. CNES.

8. F. Baron, F. Cassaing, and L. Mugnier. Alignement des pupilles d'un télescope multi-pupilles. In J.M. Chassery and C. Jutten, editors, 19ième Colloque sur le Traitement du Signal et des Images. GRETSI, September 2003.

9. F. Cassaing. Optical path difference sensors. C. $R$. Acad. Sci. Paris, Série IV, tome 2(1):87-98, January 2001.

10. R. A. Gonsalves. Phase retrieval and diversity in adaptive optics. Opt. Eng., 21(5):829-832, 1982.

11. R. G. Paxman, T. J. Schulz, and J. R. Fienup. Joint estimation of object and aberrations by using phase diversity. J. Opt. Soc. Am. A, 9(7):1072-1085, 1992.

12. R. L. Kendrick, D. S. Acton, and A. L. Duncan. Phase-diversity wave-front sensor for imaging systems. Appl. Opt., 33(27):6533-6546, 1994.

13. D. J. Lee, M. C. Roggemann, B. M. Welsh, and E. R. Crosby. Evaluation of least-squares phasediversity technique for space telescope wave-front sensing. Appl. Opt., 36:9186-9197, December 1997.

14. D.J. Lee, B.M. Welsh, and M.C. Roggemann. Diagnosing unknown aberrations in an adaptive optics system by use of phase diversity. Opt. Lett., 22(13):952954, 1997.

15. A. Blanc. Identification de réponse impulsionnelle et restauration d'images : apports de la diversité de phase. PhD thesis, Université Paris XI Orsay, July 2002.

16. A. Blanc, T. Fusco, M. Hartung, L. M. Mugnier, and G. Rousset. Calibration of NAOS and CONICA static aberrations. Application of the phase diversity technique. Astron. Astrophys., 399:373-383, 2003.

17. A. Blanc, L. M. Mugnier, and J. Idier. Marginal estimation of aberrations and image restoration by use of phase diversity. J. Opt. Soc. Am. A, 20(6):1035-1045, 2003.

18. M. G. Löfdahl and G. B. Scharmer. Wavefront sensing and image restoration from focused and defocused solar images. Astron. Astrophys., 107:243-264, 1994.

19. J. H. Seldin and R. G. Paxman. Phase-diverse speckle reconstruction of solar data. In T.J. Schulz and D.L. Snyder, editors, Image Reconstruction and Restoration, volume 2302, pages 268-280. SPIE, 1994.

20. F. Baron, F. Cassaing, A. Blanc, and D. Laubier. Cophasing a wide field multiple-aperture array by phase-diversity: influence of aperture redundancy and dilution. In M. Shao, editor, Interferometry in Space, volume 4852, Hawaii, USA, 2002. Proc. Soc. PhotoOpt. Instrum. Eng., SPIE. 